\title{
Tensor hierarchy algebras and extended geometry. Part II. Gauge structure and dynamics
}

\author{
Martin Cederwall ${ }^{a}$ and Jakob Palmkvist ${ }^{a, b}$ \\ ${ }^{a}$ Division for Theoretical Physics, Department of Physics, Chalmers University of Technology, \\ SE-412 96 Gothenburg, Sweden \\ ${ }^{b}$ Division for Algebra and Geometry, Department of Mathematical Sciences, \\ Gothenburg University and Chalmers University of Technology, \\ SE-412 96 Gothenburg, Sweden \\ E-mail: martin.cederwall@chalmers.se, jakob.palmkvist@chalmers.se
}

ABSTRACT: The recent investigation of the gauge structure of extended geometry is generalised to situations when ancillary transformations appear in the commutator of two generalised diffeomorphisms. The relevant underlying algebraic structure turns out to be a tensor hierarchy algebra rather than a Borcherds superalgebra. This tensor hierarchy algebra is a non-contragredient superalgebra, generically infinite-dimensional, which is a double extension of the structure algebra of the extended geometry. We use it to perform a (partial) analysis of the gauge structure in terms of an $L_{\infty}$ algebra for extended geometries based on finite-dimensional structure groups. An invariant pseudo-action is also given in these cases. We comment on the continuation to infinite-dimensional structure groups. An accompanying paper [1] deals with the mathematical construction of the tensor hierarchy algebras.

KeYwords: Differential and Algebraic Geometry, Gauge Symmetry, M-Theory, SpaceTime Symmetries

ARXIV EPRINT: 1908.08696 


\section{Contents}

1 Introduction 1

2 Review of extended geometry 2

2.1 Generalised diffeomorphisms and ancillary transformations 2

2.2 Dynamics 5

2.3 Gauge structure, $L_{\infty}$ and the Borcherds superalgebra $\mathscr{B} \quad 6$

$\begin{array}{lll}3 & \text { The tensor hierarchy algebra } S & 7\end{array}$

4 Ancillary transformations from $S \quad 12$

5 Dynamics 14

$\begin{array}{llr}6 & L_{\infty} \text { algebra } & 15\end{array}$

$\begin{array}{ll}6.1 & \text { Definitions and identities } \\ 6.2 & 16\end{array}$

$\begin{array}{ll}6.2 \text { Some } L_{\infty} \text { brackets } & 18\end{array}$

$\begin{array}{lll}7 & \text { Discussion } & 22\end{array}$

\section{Introduction}

Extended geometry $[2,3]$ has recently emerged as a unified framework, with double and exceptional geometry as special cases where string theory dualities are geometrised. It has been shown to have close relations to certain classes of Lie superalgebras [2-5]. The purpose of the present paper is to demonstrate how indeed the recently invented tensor hierarchy algebras (THA's) [6, 7] are to be seen as the algebraic structure responsible for and underlying extended geometry, in its most general setting. Besides unifying double geometry [8-22] and exceptional geometry [23-44], one of the advantages of the framework of extended geometry is that it opens a window to situations with infinite-dimensional structure groups [42-44]. Eventually, we would like to establish contact with the $E_{10}$ [45] and $E_{11}$ [46] proposals. In the present paper, we will however limit our attention to finitedimensional structure groups.

We consider extended geometry based on a Kac-Moody algebra $\mathfrak{g}$ of rank $r$ and a dominant integral weight $\lambda$, as defined in ref. [2]. Here, we assume $\mathfrak{g}$ to be finite-dimensional and simply laced (or at least that the Dynkin labels $\lambda_{i}$ corresponding to short roots $\alpha_{i}$ vanish). This still includes ordinary, double and (up to $r=8$ ) exceptional geometry, as well as many other, more "exotic" extended geometries. We are particularly interested in the cases where the highest root $\theta$ of $\mathfrak{g}$ satisfies $(\lambda, \theta)>1$, which implies that $\mathfrak{g}^{+}$is infinite-dimensional. As shown in ref. [2], these are exactly the cases where the generalised 
diffeomorphisms do not close into themselves, but only up to "ancillary" $\mathfrak{g}$ transformations [39, 40, 42, 47]. For this reason, they were not included in the analysis in ref. [3], where the associated $L_{\infty}$ algebra structure was derived from a Borcherds superalgebra $\mathscr{B}\left(\mathfrak{g}^{+}\right)$. In the present paper, we extend the analysis and include the cases where ancillary transformations are present by using a THA $S\left(\mathfrak{g}^{+}\right)$rather than $\mathscr{B}\left(\mathfrak{g}^{+}\right)$. Accordingly, also the study of the THA's, restricted to finite-dimensional $\mathfrak{g}^{+}$in [7], needs to be extended. This extended study is carried out in the accompanying paper [1].

Throughout the paper, all considerations concern what in the context of e.g. double or extended geometry, or the geometrisation of Ehlers symmetry, would be the "internal" sector. Most situations we describe do not have an interpretation in terms of string theory or M-theory duality, although geometrisation of any duality arising on compactification to 3 dimensions or higher is covered. Dualities in 3 dimensions generically correspond to extended geometry with the adjoint as coordinate representation. This is a subclass of the models described in this paper, but not included in the analysis of ref. [3].

The paper is organised as follows. Section 2 contains a brief review of earlier work on the gauge structure and dynamics of extended geometry in cases where $(\lambda, \theta)=1$. Section 3 describes the THA $S\left(\mathfrak{g}^{+}\right)$with focus on a particularly useful double grading. For more detail, we refer to the companion paper [1]. We then perform a detailed investigation of the ancillary transformations, as obtained from the THA, in section 4 . The dynamics is formulated by means of a pseudo-action, using structure constants of the THA, in section 5 , and the $L_{\infty}$ gauge structure is outlined in section 6 . We conclude with a discussion in section 7 , with focus on the continuation to infinite-dimensional structure algebras $\mathfrak{g}$.

The accompanying paper [1] deals with the construction of the THA's from generators and relations, and other purely algebraic aspects. In order for both papers to be reasonably self-contained, their contents have a certain overlap.

\section{Review of extended geometry}

The input for extended geometry [2] is a structure group $G$ (with Lie algebra $\mathfrak{g}$ ) together with a lowest weight "coordinate representation" (i.e., the representation of a generalised tangent vector) $R(-\lambda)$. The generalised tangent space is the lowest weight module $R(-\lambda)$, where $\lambda$ is an integral dominant weight. The structure algebra can in principle be any Kac-Moody algebra, but we will, for simplicity, take $\mathfrak{g}$ to be simply laced (or at least that $\lambda_{i}=0$ when $\alpha_{i}$ is a short root) and normalise the simple roots to have length squared 2.

In what follows, we often use an extension of $\mathfrak{g}$ to a Lie algebra $\mathfrak{g}^{+}$, which is obtained by adjoining one node, corresponding to a simple root with length squared 2, to the Dynkin diagram of $\mathfrak{g}$, with lines corresponding to the coefficients of $\lambda$ expressed in the basis of fundamental weights, see figure 1 .

\subsection{Generalised diffeomorphisms and ancillary transformations}

Generalised diffeomorphisms with infinitesimal parameter $\xi \in R(-\lambda)$, acting on a vector $V \in R(-\lambda)$, are given in terms of the generalised Lie derivative,

$$
\mathscr{L}_{\xi} V^{M}=\xi^{N} \partial_{N} V^{M}+Z_{P Q}{ }^{M N} \partial_{N} \xi^{P} V^{Q} .
$$




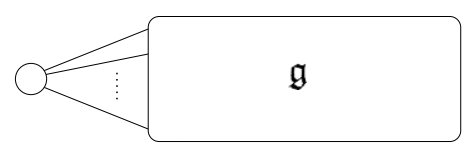

Figure 1. The Dynkin diagram for $\mathfrak{g}^{+}$.

The second term is a local $\mathfrak{g} \oplus \mathbb{R}$ transformation of $V$. The invariant tensor $Z$ has the universal expression

$$
\sigma Z=-\eta^{\alpha \beta} t_{\alpha} \otimes t_{\beta}+(\lambda, \lambda)-1
$$

where $\sigma$ is the permutation operator, $t_{\alpha M^{N}}$ representation matrices, and $\eta$ the inverse Killing metric. Here, and in the following, we use an index-free notation, where $Z$ is seen as an operator $Z: R(\lambda) \otimes R(\lambda) \rightarrow R(\lambda) \otimes R(\lambda)$. The Killing metric and its inverse will often be used to implicitly raise and lower adjoint indices. Note that the generalised Lie derivative fulfils a Leibniz rule.

Calculating the commutator of two generalised diffeomorphisms, and using the section constraint gives the result [2]

$$
\left[\mathscr{L}_{\xi}, \mathscr{L}_{\eta}\right] V=\left(\mathscr{L}_{\frac{1}{2}\left(\mathscr{L}_{\xi} \eta-\mathscr{L}_{\eta} \xi\right)}+\Sigma_{\xi, \eta}\right) V .
$$

The second term on the right hand side is a so-called ancillary transformation, which is a restricted local $\mathfrak{g}$ transformation.

The calculation leading to (2.3) uses the section constraint. This constraint ensures that any two derivatives on any field or parameter lies in a linear subspace (a section) of the minimal orbit of $R(\lambda)$ under $G$. The stability group of a section is a parabolic subgroup of $G$ containing $\mathrm{GL}(d)$, the local structure group of gravity. The section constraint reads

$$
Y \partial \otimes \partial=0
$$

where $Y=Z+1$, i.e.,

$$
\sigma Y=-\eta^{\alpha \beta} t_{\alpha} \otimes t_{\beta}+(\lambda, \lambda)-1+\sigma .
$$

The relation of the section constraint to minimal orbits was elaborated in refs. $[2,48]$. On a state ${ }^{1} \phi \in R(2 \lambda) \subset \vee^{2} R(\lambda)$, one has

$$
\eta^{\alpha \beta} t_{\alpha} \otimes t_{\beta} \phi=(\lambda, \lambda) \phi
$$

and on $\psi \in R\left(2 \lambda-\alpha_{i}\right) \subset \wedge^{2} R(\lambda)$, where $\left(\lambda, \alpha_{i}\right)=1$,

$$
\eta^{\alpha \beta} t_{\alpha} \otimes t_{\beta} \psi=((\lambda, \lambda)-2) \psi
$$

These relations are straightforward to derive using the eigenvalues of the quadratic Casimir operator in the respective representations. The representations $R(2 \lambda)$ (symmetric) and $\bigoplus_{i: \lambda_{i}=1} R\left(2 \lambda-\alpha_{i}\right)$ (antisymmetric) are the representations in $\otimes^{2} R(\lambda)$ which are annihilated by $Y$.

\footnotetext{
${ }^{1}$ We use the notation $\vee$ for the symmetrised and $\wedge$ for the anti-symmetrised product.
} 
The ancillary transformation $\Sigma_{\xi, \eta}$ has the generic form [2]

$$
\Sigma_{\xi, \eta} V^{M}=\frac{1}{2} S^{\alpha}{ }_{P Q}{ }^{R S} \xi^{P} \partial_{R} \partial_{S} \eta^{Q} t_{\alpha N^{M}} V^{N}-(\xi \leftrightarrow \eta),
$$

the tensor $S$ being given by the simple expression ${ }^{2}$

$$
S^{\alpha}{ }_{M N}^{P Q}=\left(f^{\alpha \beta \gamma} t_{\beta} \otimes t_{\gamma}+t^{\alpha} \otimes \mathbb{1}-\mathbb{1} \otimes t^{\alpha}\right)_{M N}\langle P Q\rangle .
$$

The notation $\langle P Q\rangle$ means projection on $R(2 \lambda)$ or $R(-2 \lambda)$. Later, we will also denote the projection on the antisymmetric $\bigoplus_{i: \lambda_{i}=1} R\left(2 \lambda-\alpha_{i}\right)$ or its conjugate by a curly bracket $\{M N\}$. The tensor $S$ is antisymmetric in its lower indices, and obeys $S^{\alpha}{ }_{\{M N\}}{ }^{P Q}=0$.

The tensor $S$ vanishes when $\mathfrak{g}$ is a finite-dimensional algebra and $\lambda$ is a weight with $(\lambda, \theta)=1$, i.e., a fundamental weight dual to a simple root with Coxeter label 1. In ref. [2] we gave the following list of cases where ancillary transformations are absent:

- $\mathfrak{g}=A_{r}, \lambda=\Lambda_{p}, p=1, \ldots, r$ ( $p$-form representations $)$

- $\mathfrak{g}=B_{r}, \lambda=\Lambda_{1}$ (the vector representation);

- $\mathfrak{g}=C_{r}, \lambda=\Lambda_{r}$ (the symplectic-traceless $r$-form representation);

- $\mathfrak{g}=D_{r}, \lambda=\Lambda_{1}, \Lambda_{r-1}, \Lambda_{r}$ (the vector and spinor representations);

- $\mathfrak{g}=E_{6}, \lambda=\Lambda_{1}, \Lambda_{5}$ (the fundamental representations);

- $\mathfrak{g}=E_{7}, \lambda=\Lambda_{1}$ (the fundamental representation)

In many of these cases, $\mathfrak{g}^{+}$is finite-dimensional, but the list also contains cases where $\mathfrak{g}^{+}$ is infinite-dimensional:

- $\mathfrak{g}=A_{7}, \lambda=\Lambda_{4}$

- $\mathfrak{g}=A_{r}, r \geqslant 8, \lambda=\Lambda_{p}, p=3, \ldots, r-2$

- $\mathfrak{g}=C_{r}, r \geqslant 4, \lambda=\Lambda_{r}$;

- $\mathfrak{g}=D_{r}, r \geqslant 8, \lambda=\Lambda_{r-1}, \Lambda_{r}$

Assuming that $\mathfrak{g}$ is simply laced, the presence of ancillary transformations implies that $\mathfrak{g}^{+}$is infinite-dimensional. We will not consider the cases where $\mathfrak{g}$ is not simply laced further in the present paper, but the construction should work as long as $\lambda$ is orthogonal to all short roots.

\footnotetext{
${ }^{2}$ In ref. [2], the projection was not included in the definition of the tensor $S$. In addition, there is an apparent sign difference to the expression given there; this is due to the change of conventions for the $t_{\alpha}$ 's.
} 


\subsection{Dynamics}

In ref. [2], a (pseudo-)action ${ }^{3}$ was given, encoding the dynamics of any extended geometry in the absence of ancillary transformations, and in the special cases where the coordinate representation is the adjoint.

The generalised metric $G_{M N}$ parametrises the coset $G / K \times \mathbb{R}$. It is convenient, in order to write an action without reference to any "external" dimensions, to let $G_{M N}$ transform as a tensor density with weight $-2 w=1-2(\lambda, \lambda)$. The generalised metric induces a preferred involution on $\mathfrak{g}$ through $t_{\alpha M^{N}} \mapsto-t_{\alpha M}^{\star}{ }^{N}=-\left(G^{-1} t_{\alpha} G\right)^{N}{ }_{M}$, i.e., $t^{\star}=G t^{t} G^{-1}$.

Define

$$
\left(\partial_{M} G G^{-1}\right)_{N}{ }^{P}=\Pi_{\alpha M} t^{\alpha}{ }_{N}^{P}+\Pi_{M} \delta_{N}^{P}
$$

(this decomposition follows from $G$ being a group element in $G \times \mathbb{R}$ ). Checking the transformation of a Lagrangian up to total derivatives, only the inhomogeneous transformations $\Delta_{\xi} \equiv \delta_{\xi}-\mathscr{L}_{\xi}$ are needed. They are

$$
\begin{aligned}
\Delta_{\xi} \Pi_{M} & =-2 w \partial_{M} \partial_{N} \xi^{N}, \\
\Delta_{\xi} \Pi_{\alpha M} & =\left(t_{\alpha}+t_{\alpha}^{\star}\right)_{P}{ }^{N} \partial_{M} \partial_{N} \xi^{P} .
\end{aligned}
$$

It was then shown that the combination of terms

$$
\mathscr{L}_{0}=\frac{1}{2} A-B-2 C-\frac{(\lambda, \lambda)}{(\lambda, \lambda)-\frac{1}{2}} D,
$$

with

$$
\begin{aligned}
& A=G^{M N} \eta^{\alpha \beta} \Pi_{\alpha M} \Pi_{\beta N}, \\
& B=G^{P Q} t^{\alpha}{ }_{P}{ }^{M} t^{\beta}{ }^{N} \Pi_{\alpha N} \Pi_{\beta M}, \\
& C=\left(G^{-1} t^{\alpha}\right)^{M N} \Pi_{M} \Pi_{\alpha N}, \\
& D=G^{M N} \Pi_{M} \Pi_{N},
\end{aligned}
$$

has the inhomogeneous transformation, up to a total derivative,

$$
\Delta_{\xi} \mathscr{L}_{0}=-2 S^{\alpha}{ }_{P Q}{ }^{M N} G^{P S} \Pi_{\alpha S} \partial_{M} \partial_{N} \xi^{Q}
$$

The calculation relies on the section condition, both its symmetric part on the two derivatives on $\xi$ and its general form on $\Pi$ and one of the derivatives, in order to cancel terms produced by the $\sigma$ term in the $Y$ tensor, and thereby relating terms with adjoint and scalar parts of $\Pi$. The weight of each derivative is $-(\lambda, \lambda)+1$ and that of an inverse metric $2(\lambda, \lambda)-1$, so each term in the Lagrangian has weight 1 . This is the correct weight for a density $\omega$ so that being a divergence $\omega=\partial_{M} v^{M}$ of a vector density $v$ is a covariant property [2]. The Lagrangian $\mathscr{L}_{0}$ thus describes the dynamics in the absence of ancillary transformations.

\footnotetext{
${ }^{3}$ We are reluctant to use the term action as long as the section constraint needs to be imposed by hand.
} 


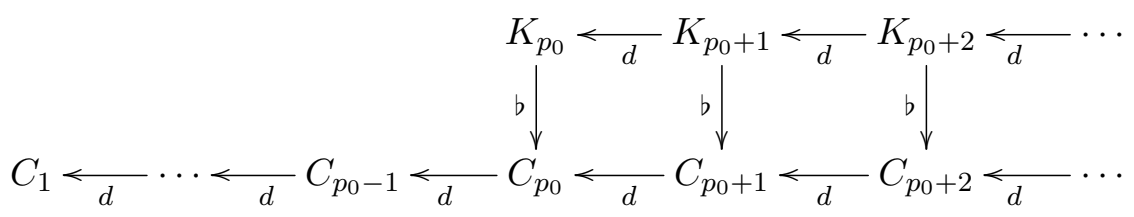

Table 1. The typical structure of the action of the 1-bracket between the ghost modules, with ancillary ghosts appearing from level $p_{0} \geqslant 1$.

\subsection{Gauge structure, $L_{\infty}$ and the Borcherds superalgebra $\mathscr{B}$}

In refs. [3, 4], the generalised tangent space was identified with a subspace of a Borcherds superalgebra, here denoted $\mathscr{B}\left(\mathfrak{g}^{+}\right)$, or simply just $\mathscr{B}$. This superalgebra is obtained by a double extension of the structure algebra $\mathfrak{g}$, first to $\mathfrak{g}^{+}$by a node corresponding to the weight $\lambda$, attached to node $i$ in the Dynkin diagram of $\mathfrak{g}$ with a number of lines equalling the Dynkin labels $\lambda_{i}$ of $\lambda$, and then by a "grey" node attached with a single line to the first extending node. The simple root corresponding to the grey node is an odd null root. The resulting Dynkin diagram is shown as the right diagram in figure 2. The left diagram in the same figure is equivalent. The Borcherds superalgebra $\mathscr{B}$ has a consistent $(\mathbb{Z} \times \mathbb{Z})$-grading, corresponding to the two leftmost nodes in the left diagram. We denote the corresponding grades by $p$ and $q$ (where $q$ corresponds to the leftmost node) and refer to them as level and height, respectively.

Our notation for the basis elements in the local part of the algebra with respect to the level $p$ is given in table 2. At height $q=0$, we have the subalgebra $\mathscr{B}(\mathfrak{g}) \oplus \mathbb{R}$, and at $p=q$ the subalgebra $\mathfrak{g}^{+} \oplus \mathbb{R}$. Elements at a given $(\mathbb{Z} \times \mathbb{Z})$-grade form $\mathfrak{g}$-modules. The modules at even and odd $p+q$ belong to the even and odd parts of the Lie superalgebra, respectively. We refer to refs. [1,3] for more detail.

The subspace where the vector fields live is the one at $(p, q)=(1,0)$, with basis $E_{M}$, but in order to describe the gauge structure it is convenient to define a generalised Lie derivative for any pair of elements $A$ and $B$ at $p \geqslant 0$ and $q=0$, which are also allowed to have odd (fermionic) components. The generalised Lie derivative is then constructed $[2-5]$ as

$$
\mathscr{L}_{A} B=\delta_{p_{A}, 1}\left(\left[\left[A, F^{b M}\right], \partial_{M} B\right]+(-1)^{|B|}\left[\left[\partial_{M} A^{\sharp}, F^{b M}\right], B\right]\right),
$$

where $|B|$ is the total statistics of $B$ and $p_{A}$ is the level of $A$. The bracket $[\cdot, \cdot]$ is that of the Borcherds superalgebra $\mathscr{B}\left(\mathfrak{g}^{+}\right)$(we use this notation also for the symmetric bracket between two totally fermionic elements).

The ghosts $C_{p}$ reside at height $q=0$, and the ancillary ghosts $K_{p}$ at $q=1$, see table 1. They can be combined into $C=\sum_{p=1}^{\infty} C_{p}$ and $K=\sum_{p=p_{0}}^{\infty} K_{p}$. Both the ghosts and ancillary ghosts are totally bosonic, $|C|=|K|=0$. The ghost number is $p+q$. Note that $p_{0} \geqslant 1$, which means that the Borcherds superalgebra can not accommodate ancillary transformations, corresponding to ancillary ghosts with ghost number 1, which would reside at $(p, q)=(0,1)$ and transform as the adjoint. This will be remedied by the introduction of the tensor hierarchy algebras. 
We denote $C=c+C^{\prime}$, where $c=C_{1}$. In ref. [3] it was shown that the ghosts satisfy an $L_{\infty}$ algebra [49-52]. The full list of non-vanishing brackets is:

$$
\begin{aligned}
\llbracket C^{\prime} \rrbracket & =d C^{\prime}, \\
\llbracket K \rrbracket & =d K+K^{b}, \\
\llbracket C^{n} \rrbracket & =k_{n}\left((\operatorname{ad} C)^{n-2}\left(\mathscr{L}_{C} C+X_{C} C\right)+\sum_{i=0}^{n-3}(\operatorname{ad} C)^{i} R_{C}(\operatorname{ad} C)^{n-i-3} \mathscr{L}_{C} C\right) \\
\llbracket C^{n-1}, K \rrbracket & =\frac{k_{n}}{n}\left((\operatorname{ad} C)^{n-2} \mathscr{L}_{C} K+\sum_{i=0}^{n-3}(\operatorname{ad} C)^{i} \operatorname{ad} K(\operatorname{ad} C)^{n-i-3} \mathscr{L}_{C} C\right),
\end{aligned}
$$

where the coefficients $k_{n}$ have the universal model-independent expression in terms of Bernoulli numbers

$$
k_{n+1}=\frac{2^{n} B_{n}^{+}}{n !}, \quad n \geqslant 1 .
$$

We here use the convention that the brackets are graded symmetric, all brackets carry ghost number -1 , and " $C$ " "represents " $C, C, \ldots, C$ ". All non-vanishing brackets except the 1-bracket contain at least one level 1 ghost $c$. No brackets contain more than one ancillary ghost.

The meaning of the symbols in eq. (2.16) will be explained in detail in section 6. Here, it suffices to mention that $X$ and $R$ represent ancillary contributions, and that the nilpotent "derivatives" $d$ and $b$ act to the left and down, respectively (see table 1), in the double grading of table 2 .

\section{The tensor hierarchy algebra $S$}

When $\mathfrak{g}=A_{n-1}$, i.e., when extended geometry is geometry with structure group GL $(n)$, the tensor hierarchy algebras are $W\left(A_{n-1}\right)=W(n)$, and $S\left(A_{n-1}\right)=S(n)$, the finitedimensional non-contragredient superalgebras of Cartan type in Kac's classification [53]. The definition of these superalgebras in terms of generators and relations derived from a Dynkin diagram - in spite of them being non-contragredient — was given in ref. [7]. This construction is extended in the accompanying paper [1] to $W\left(\mathfrak{g}^{+}\right)$and $S\left(\mathfrak{g}^{+}\right)$for finite-dimensional $\mathfrak{g}$.

Here we will focus on $S=S\left(\mathfrak{g}^{+}\right)$, where $\mathfrak{g}^{+}$is obtained, as explained above, by adding one node to a finite-dimensional Lie algebra $\mathfrak{g}$, which is the structure algebra of the extended geometry under consideration. The superalgebra $S$ turns out to be appropriate for the description of extended geometry; the extra elements in $W=W\left(\mathfrak{g}^{+}\right)$are not required. Both $W$ and $S$ can be described by the same Dynkin diagram as $\mathscr{B}$, but with different assignment of generators and relations [1]. This doubly extended diagram was described in the preceding section and is given schematically in figure 2 .

For the construction of gauge transformations etc. (and ultimately the $L_{\infty}$ algebra), we use the same double grading as for $\mathscr{B}$, splitting the superalgebra into finite-dimensional representations of $\mathfrak{g}$, labelled by two integers $(p, q)$. The subalgebra $W(\mathfrak{g}) \subset S\left(\mathfrak{g}^{+}\right)$is on 

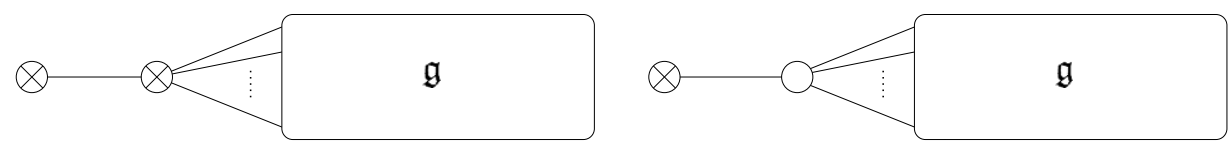

Figure 2. Two equivalent Dynkin diagrams for $\mathscr{B}\left(\mathfrak{g}^{+}\right), W\left(\mathfrak{g}^{+}\right)$and $S\left(\mathfrak{g}^{+}\right)$.

\begin{tabular}{|c|c|c|c|c|}
\hline & $p=-1$ & & $p=0$ & $p=1$ \\
\hline$q=1$ & & & $f_{0}$ & $E_{M}^{\sharp}$ \\
\hline$q=0$ & $F^{M}$ & & $T_{\alpha} \tilde{k}$ & $E_{M}$ \\
\hline$q=-1$ & $F^{b M}$ & & $e_{0}$ & \\
\hline
\end{tabular}

Table 2. Basis elements for $\mathscr{B}$ at $p=-1,0,1$.

the line $q=0$, and the Lie subalgebra $\mathfrak{g}^{+} \subset S\left(\mathfrak{g}^{+}\right)$is on the diagonal $p=q$ (generically together with other elements).

A way of deriving the content of $S$ is to note that the generators $E_{M}$, which form a basis for $R_{1}=R(-\lambda)$, have a covariant Serre relation in $R(-2 \lambda)$, so that the anticommutator $\left[E_{M}, E_{N}\right]$ lies in $R_{2}=\vee^{2} R_{1} \ominus R(-2 \lambda)$. Any element at $(p, q)=(-1,0)$ must respect the ideal generated by the Serre relations. This allows for the introduction of generators $\Phi_{\alpha}{ }^{M}$ with anticommutators

$$
\left[E_{N}, \Phi_{\alpha}^{M}\right]=\varphi^{\beta}{ }_{N, \alpha}^{M} T_{\beta}
$$

where $\varphi$ is a linear combination of projection operators on the irreducible modules appearing in $\varphi$. They respect the ideal in $R(-2 \lambda)$ if

$$
t_{\beta\left\langle M^{P}\right.} \varphi^{\beta}{ }_{N\rangle, \alpha}{ }^{Q}=\left(t_{\beta} \otimes \varphi^{\beta}{ }_{\alpha}\right)_{\langle M N\rangle}{ }^{P Q}=0,
$$

or equivalently, $\left(\phi^{\alpha}{ }_{\beta} \otimes t^{\beta}\right)_{M N}\langle P Q\rangle=0$. where $\langle M N\rangle$ is projection on $R( \pm 2 \lambda)$. Eq. (3.2) is the condition for the representation of the embedding tensor, or the "big torsion representation" ${ }^{4}$ The content of $\Phi$ in terms of irreducible representations can be determined by decomposing $\mathfrak{g}$ into levels $\ell$ with respect to $\lambda$ (so that the level of a root $\alpha$ is $(\lambda, \alpha)$ ) [1]. If we then let $H_{0}$ be the set of highest roots at level 0 and $L_{\ell}$ the set of lowest roots at level $\ell$, then the content of $\Phi$ is

$$
(N-1) R(\lambda) \oplus \bigoplus_{\gamma \in H_{0}} R(\lambda+\gamma) \oplus \bigoplus_{\ell=2}^{(\lambda, \theta)} \bigoplus_{\beta \in L_{\ell}} R(\lambda-\beta),
$$

where $N$ is the number of non-vanishing Dynkin labels of $\lambda$.

\footnotetext{
${ }^{4}$ Although we have not performed a complete analysis, we have noted that in cases when $\lambda$ is attached to a short root, there is typically no solution to this algebraic condition.
} 


\begin{tabular}{|c|c|c|c|}
\hline & $p=-1$ & $p=0$ & $p=1$ \\
\hline$q=2$ & & & $L_{\alpha M}^{\sharp}$ \\
\hline$q=1$ & $\Phi_{\alpha}^{\sharp M}$ & $T_{\alpha}^{\sharp}$ & $E_{M}^{\sharp} \quad L_{\alpha M}$ \\
\hline$q=0$ & $\Phi_{\alpha}{ }^{M} \quad H^{M}$ & $T_{\alpha} \tilde{k}$ & $E_{M}$ \\
\hline$q=-1$ & $H^{\mathrm{bM}}$ & $e_{0}$ & \\
\hline
\end{tabular}

Table 3. Basis elements for $S$ at $p=-1,0,1$.

\begin{tabular}{|c|c|c|c|}
\hline & $p=-1$ & $p=0$ & $p=1$ \\
\hline$q=2$ & & & $L_{\alpha M}^{\sharp}$ \\
\hline$q=1$ & $\Phi_{\alpha}^{\sharp M} \quad G^{\sharp M}$ & $f_{0} \quad T_{\alpha}^{\sharp}$ & $E_{M}^{\sharp} \quad L_{\alpha M}$ \\
\hline$q=0$ & $F^{M} \quad \Phi_{\alpha}^{M} \quad G^{M}$ & $\begin{array}{lll}k & T_{\alpha} & \widetilde{k}\end{array}$ & $E_{M}$ \\
\hline$q=-1$ & $F^{b M}$ & $e_{0}$ & \\
\hline
\end{tabular}

Table 4. Basis elements for $W$ at $p=-1,0,1$.

The difference at $p=1$ between tables 2 and 3 illustrates the shortcoming of $\mathscr{B}\left(\mathfrak{g}^{+}\right)$, namely that it does not contain a module $\widetilde{R}_{1}$, and thus is unable to encode ancillary transformations (ancillary ghosts with ghost number 1). The THA's, on the other hand, contain $\widetilde{R}_{1}$ precisely when ancillary transformations occur - the corresponding generators are $L_{\alpha M}$ and $L_{\alpha M}^{\sharp}$ in table 3. There are of course further differences. At $p=0, q=1$, the THA has an adjoint element, which is where the ancillary ghost will reside. At $p=-1$, there is also room for generalised torsion. We comment on the inclusion of dynamical variables in the discussion in section 7 .

In a single grading with respect to the leftmost node in the second diagram of figure 2 , i.e., where the grading where each grade $n=p-q$ forms a $\mathfrak{g}^{+}$-module, the positive levels of $W$ agree with those of the corresponding Borcherds superalgebra $\mathscr{B}$. Also the positive levels of $S$ essentially agree with those of $\mathscr{B}$, but in some cases, there may be an issue of further ideals arising at positive levels in the definition of the algebra $S$ [1]. Up to such ideals (further discussed in ref. [1]), both $S$ and $\mathscr{B}$ are subalgebras of $W$, with the grading shown in table 4 . The elements in the subalgebras denoted by the same symbols as in $W$ are identified. In addition, the elements $F^{b}$ and $H^{b}$ are the same, although $F$ and $H$ are 
not; $H$ is the linear combination ${ }^{5} H^{M}=F^{M}+\frac{1}{(\lambda, \lambda)} G^{M}$. The criterion determining this combination (apart from $H^{b}=F^{b}$ ) is that the bracket $\left[E_{M}, H^{N}\right]$ may contain $\widetilde{k}$ but not $k$. The lowering operation is defined as $A^{b}=-\left[A, e_{0}\right]$ on any element. The raising operator is defined so that $A^{\sharp}=0$ for an upper element in a pair, and $A^{b \sharp}+A^{\sharp b}=A$. Although we use the same notation for raising and lowering as for the Borcherds superalgebra in ref. [2], these are different operations (which is illustrated by $H$ and $H^{b}$ above). The ones in the Borcherds superalgebra are inherited from $W$, where raising is not defined at $p=0$. On the other hand, in $S$, raising and lowering are well defined operations at level $p=0$, but it is not completely clear how to define the raising operator in general.

We refer to ref. [1] for the mathematical details concerning the definition and construction of $S$. For the class of algebras considered here, the representation content of $\widetilde{R}_{1}$ can be explicitly determined,

$$
\widetilde{R}_{1}=\bigoplus_{\ell=2}^{(\lambda, \theta)} \bigoplus_{\beta \in L_{\ell}} R(-(\lambda-\beta)) .
$$

Note that the earlier criterion for the absence of ancillary transformations [2], namely $(\lambda, \theta)=1$, implies that the grading is a 3 -grading with $\ell=-1,0,1$, and the sum in (3.4) becomes empty. The simplest class of examples with non-vanishing $\widetilde{R}_{1}$ is when $\lambda=\theta$, so that $R_{1}$ is the adjoint of $\mathfrak{g}$. Then $(\lambda, \theta)=2$, and the sum contains a single irreducible representation, the singlet.

It is a peculiar property of the THA's, that when they are the super-extension of an infinite-dimensional Kac-Moody algebra (here, $\mathfrak{g}^{+}$), already the level $n=0$ (in the double grading, $p-q=0$ ) contains elements beyond the adjoint of $\mathfrak{g}^{+}$[48]. This property follows [1] from the natural generalisation of the identities for the generators associated to the Dynkin diagram given in ref. [7]. It also provides exactly the structure needed for the description of the ancillary transformations, which will be explained in the following section. See ref. [1] for more detail.

Some non-trivial brackets in $S$ include

$$
\begin{aligned}
{\left[\widetilde{k}, E_{M}\right] } & =(1-(\lambda, \lambda)) E_{M}, \\
{\left[\widetilde{k}, e_{0}\right] } & =-e_{0}, \\
{\left[E_{M}, H^{N}\right] } & =-\left(1-\frac{1}{(\lambda, \lambda)}\right) t^{\alpha}{ }_{M}{ }^{N} T_{\alpha}+\delta_{M}^{N} \widetilde{k}, \\
{\left[E_{M}, H^{b N}\right] } & =\delta_{M}^{N} e_{0}, \\
{\left[E_{M}^{\sharp}, H^{b N}\right] } & =-t^{\alpha}{ }_{M}{ }^{N} T_{\alpha}+\delta_{M}^{N} \widetilde{k}, \\
{\left[E_{M}^{\sharp}, H^{N}\right] } & =-\frac{1}{(\lambda, \lambda)} t^{\alpha} M^{N} T_{\alpha}^{\sharp}, \\
{\left[T_{\alpha}^{\sharp}, H^{b M}\right] } & =t_{\alpha N}{ }^{M} H^{N}+\Phi_{\alpha}{ }^{M}, \\
{\left[T_{\alpha}^{\sharp}, E_{M}\right] } & =t_{\alpha M}{ }^{N} E_{N}^{\sharp}+L_{\alpha M} .
\end{aligned}
$$

\footnotetext{
${ }^{5}$ The special case $(\lambda, \lambda)=1$ requires a different treatment. In that case $W(\mathfrak{g}) \not \subset S\left(\mathfrak{g}^{+}\right)$. This happens for $\mathfrak{g}=D_{r}, \lambda=\Lambda_{1}$, i.e., for double geometry. Since no ancillary ghosts appear, we have not investigated this case further.
} 
The last two of these can be taken as definitions of $\Phi$ and $L$, containing the modules (3.3) and (3.4), respectively. We also postulate

$$
\begin{aligned}
{\left[E_{N}, \Phi_{\alpha}{ }^{M}\right] } & =\varphi^{\beta}{ }_{N, \alpha}{ }^{M} T_{\beta}, \\
{\left[L_{\alpha M}, H^{b N}\right] } & =\ell_{\alpha M}{ }^{\beta N} T_{\beta} .
\end{aligned}
$$

These relations serve to define the $\mathfrak{g}$-invariant tensors $\varphi$ and $\ell$, which by construction are some linear combinations of projectors on the irreducible modules in $\Phi$ and $L$.

Using the commutators, one can also check explicitly that $\Phi$ respects the ideal $\bigoplus_{i: \lambda_{i}=1} R\left(-\left(2 \lambda-\alpha_{i}\right)\right)$ in $\left[E_{M}^{\sharp}, E_{N}^{\sharp}\right]$. The condition becomes

$$
L_{\beta\{N}^{\sharp} \varphi_{P\}, \alpha}^{\beta}=0 .
$$

This is automatically satisfied, since the highest representations in $\varphi$ and $\ell$ are $R\left(\lambda+\gamma_{0}\right)$ and $R\left(\lambda-\beta_{2}\right)$, where $\gamma_{0}$ is a highest root at level 0 . The tensor product can not contain $R\left(2 \lambda-\alpha_{i}\right)$.

Consider the Jacobi identity between $T_{\alpha}^{\sharp}, E_{M}$ and $H^{b N}$. This turns out to be the only non-trivial Jacobi identity within the local superalgebra at $p=-1,0,1$, in the sense that all others can be obtained from it by raising and lowering operations. A short calculation leads to the necessary and sufficient condition for this Jacobi identity to be fulfilled:

$$
\varphi^{\beta}{ }_{M, \alpha}{ }^{N}-\ell_{\alpha M}{ }^{\beta N}=\delta_{\alpha}^{\beta} \delta_{M}^{N}-f_{\alpha}{ }^{\beta \gamma} t_{\gamma M}{ }^{N}-\frac{1}{(\lambda, \lambda)}\left(t^{\beta} t_{\alpha}\right)_{M^{N}} \equiv Q_{\alpha M}{ }^{\beta N},
$$

i.e.,

$$
\varphi_{\alpha}^{\beta}-\ell_{\alpha}{ }^{\beta}=\delta_{\alpha}^{\beta}-f_{\alpha}{ }^{\beta \gamma} t_{\gamma}-\frac{1}{(\lambda, \lambda)} t^{\beta} t_{\alpha} \equiv Q_{\alpha}{ }^{\beta} .
$$

If we now make use of the algebraic condition (3.2) on $\varphi$, the part of this relation only involving $\ell$ becomes, using the section constraint,

$$
\begin{aligned}
\ell_{\beta M}{ }^{\alpha\langle P} t^{\beta}{ }_{N}{ }^{Q\rangle} & =f^{\alpha}{ }_{\beta \gamma} t^{\beta}{ }_{M}\left\langle P t^{\gamma}{ }_{N}{ }^{Q\rangle}+t^{\alpha}{ }_{M}\left\langle P \delta_{N}^{Q\rangle}-\delta_{M}^{\langle P} t^{\alpha}{ }_{N}{ }^{Q\rangle}\right.\right. \\
& =\left(f^{\alpha}{ }_{\beta \gamma} t^{\beta} \otimes t^{\gamma}+t^{\alpha} \otimes \mathbb{1}-\mathbb{1} \otimes t^{\alpha}\right)_{M N}{ }^{\langle P Q\rangle} .
\end{aligned}
$$

We recognise the right hand side as the $S$ tensor of eq. (2.9), and thus

$$
\left(\ell_{\beta}{ }^{\alpha} \otimes t^{\beta}\right)_{M N}\langle P Q\rangle=S^{\alpha}{ }_{M N}{ }^{P Q} .
$$

The tensor $S$ is antisymmetric in its lower indices. In addition, it satisfies $S_{\{M N\}} P Q=0$, thanks to the identity

$$
S^{\alpha}{ }_{M N}^{P Q}=\left(\frac{1-\sigma}{2} Y\left(\mathbb{1} \otimes t^{\alpha}\right)\right)_{M N}\langle P Q\rangle .
$$

We would ideally like to show that there always is a solution of this form to eq. (3.9). This follows from the existence of the THA as defined in ref. [1], but seems surprisingly difficult to prove in a more direct manner, only using representation theory for $\mathfrak{g}$. The 
difficulty with analysing this equation lies in the translation between the projectors on irreducible representations in adj $\otimes R(\lambda)$ of the types $P_{\alpha M^{\beta N}}$ and $P^{\beta}{ }_{M, \alpha}{ }^{N}$, used to characterise $\ell$ and $\varphi$, respectively, which are not known explicitly (in any useful form) in the general case.

In ref. [1], we discuss the remarkable identity (3.9) in more detail, and show that the matrix $Q$ on the right hand side in a certain sense has corank 2, which makes the solution in terms of $\varphi$ and $\ell$ possible for any integrable highest weight representation of a finitedimensional simply laced $\mathfrak{g}$. Some highly non-trivial examples of this relation are also given in ref. [1].

\section{Ancillary transformations from $S$}

We can perform the calculation of the ancillary term in the commutator of two generalised diffeomorphisms with the expressions for the derived brackets directly in terms of the superalgebra brackets. The expression for the generalised Lie derivative is identical to the one using the Borcherds superalgebra [3] (but there the ancillary transformations could not be derived in terms of the superalgebra brackets).

Let

$$
\mathscr{L}_{\xi} V=\left[\left[\xi, H^{b M}\right], \partial_{M} V^{\sharp}\right]-\left[\left[\partial_{M} \xi^{\sharp}, H^{b M}\right], V\right],
$$

where $\xi$ has bosonic components, and where $\xi=\xi^{M} E_{M}, V=V^{M} E_{M}$. Consider the $\xi \partial^{2} \eta$ terms in $\left[\mathscr{L}_{\xi}, \mathscr{L}_{\eta}\right] V-\mathscr{L}_{\frac{1}{2}\left(\mathscr{L}_{\xi} \eta-\mathscr{L}_{\eta} \xi\right)} V$. They become

$$
\begin{aligned}
- & {\left[\left[\xi, H^{b M}\right],\left[\left[\partial_{M} \partial_{N} \eta^{\sharp}, H^{b N}\right], V\right]^{\sharp}\right] } \\
& +\frac{1}{2}\left[\left[\left[\left[\xi, H^{b M}\right], \partial_{M} \partial_{N} \eta^{\sharp}\right]^{\sharp}, H^{b N}\right], V\right] \\
& +\frac{1}{2}\left[\left[\left[\left[\partial_{M} \partial_{N} \eta^{\sharp}, H^{b M}\right], \xi\right]^{\sharp}, H^{b N}\right], V\right]-(\xi \leftrightarrow \eta) .
\end{aligned}
$$

In the last term, we pull out the $H^{\mathrm{b} M}$ at the price of a term with $\left[\xi, H^{b M}\right]=\xi^{M} e_{0}$. Then this term cancels the first two terms, and the remainder is

$$
-\frac{1}{2}\left[\left[\left[\left[\xi, \partial_{M} \partial_{N} \eta^{\sharp}\right], H^{b M}\right]^{\sharp}, H^{b N}\right], V\right]-(\xi \leftrightarrow \eta) .
$$

In the first step, the transformation parameter becomes

$$
\begin{aligned}
- & \frac{1}{2}\left[\left[\left[\xi, \partial_{M} \partial_{N} \eta^{\sharp}\right], H^{b M}\right]^{\sharp}, H^{b N}\right]-(\xi \leftrightarrow \eta) \\
& =-\frac{(\lambda, \lambda)}{2((\lambda, \lambda)-1)}\left[\left[\left[\xi, \partial_{M} \partial_{N} \eta^{\sharp}\right], H^{b M}\right], H^{N}\right]-(\xi \leftrightarrow \eta), \\
& =-\frac{(\lambda, \lambda)}{2((\lambda, \lambda)-1)}\left[\left[\left[\xi, \partial_{M} \partial_{N} \eta^{\sharp}\right], H^{M}\right], H^{b N}\right]-(\xi \leftrightarrow \eta),
\end{aligned}
$$

where the prefactor is to compensate for the factor in front of the $T$ term in $[E, H]$, and where potential $\widetilde{k}$ terms have been disregarded (they are easily shown to vanish). The two 
right hand sides of this equation can be calculated explicitly. In the Borcherds superalgebra, they give the same result (there, the prefactor is absent). In $S$, they give "different" expressions. The first one is identical to the Borcherds algebra calculation: roughly speaking, $\left[B_{M N}^{b}, H^{b M}\right]$ gives only $R_{1}$, but the second one goes through an intermediate $\widetilde{R}_{1}$ : $\left[B_{M N}^{b}, H^{M}\right]$ gives a combination of $R_{1}$ and $\widetilde{R}_{1}$. We use $\xi=\xi^{M} E_{M}$ etc. and calculate the expression contracting $\xi^{P} \partial_{M} \partial_{N} \eta^{Q}$. A straightforward calculation, using the section constraint, gives the two alternative expressions

$$
\begin{aligned}
& -\frac{(\lambda, \lambda)}{2((\lambda, \lambda)-1)}\left(\left[\left[\left[E_{P}, H^{b M}\right], E_{Q}^{\sharp}\right], H^{N}\right]+\left[\left[E_{P},\left[E_{Q}^{\sharp}, H^{b M}\right]\right], H^{N}\right]\right) \\
& \quad \approx \frac{1}{2}\left(2 \delta_{[P}^{M} t_{Q]}^{\alpha} T_{\alpha}-f_{\gamma}^{\alpha \beta} t_{P}^{\alpha M} t_{Q}^{\beta} T_{\gamma}\right)
\end{aligned}
$$

and

$$
\begin{aligned}
- & \frac{(\lambda, \lambda)}{2((\lambda, \lambda)-1)}\left(\left[\left[\left[E_{P}, H^{M}\right], E_{Q}^{\sharp}\right], H^{b N}\right]+\left[\left[E_{P},\left[E_{Q}^{\sharp}, H^{M}\right]\right], H^{b N}\right]\right) \\
\approx & \frac{(\lambda, \lambda)}{2((\lambda, \lambda)-1)}\left(2 \delta_{[P}^{M} t_{Q]}^{\alpha} T_{\alpha}-f^{\alpha \beta}{ }_{\gamma}^{\alpha} t_{P}^{\alpha} t_{Q}^{\beta N} T_{\gamma}\right) \\
& +\frac{1}{2((\lambda, \lambda)-1)} \ell_{\alpha P}{ }^{\beta M} t_{Q}^{\alpha} T_{\beta}
\end{aligned}
$$

respectively (the " $\approx$ " sign denotes equality when the indices are section-projected $\langle M N\rangle$ and antisymmetrised $[P Q]$ ). We recognise the $S$ tensor in the first expression. The algebra now identifies the result obtained by going through $R_{1}$ with the one obtained by going through $\widetilde{R}_{1}$, and the result becomes

$$
-\frac{1}{2} \ell_{\alpha P}{ }^{\beta M} t_{Q}^{\alpha N^{N}} T_{\beta}
$$

Unfortunately, the above calculation does not work for $(\lambda, \lambda)=1$, probably because the $q=0$ subalgebra then is not $W(\mathfrak{g})$, but quite degenerate. This does not exclude that the superalgebra $S\left(D_{r+1}\right)$ provides a good description. There, ancillary transformations are absent.

It is straightforward to show by explicit calculation that the ancillary transformation also can be expressed as

$$
\begin{aligned}
\Sigma & =-\frac{1}{2}\left[\left[\left[\xi^{\sharp}, \partial_{M} \partial_{N} \eta^{\sharp}\right], H^{b M}\right], H^{b N}\right]-(\xi \leftrightarrow \eta) \\
& =-X_{\xi}^{b} \eta+X_{\eta}^{b} \xi .
\end{aligned}
$$

The innermost bracket $\left[\xi^{\sharp}, \partial_{M} \partial_{N} \eta^{\sharp}\right]$ is in $\widetilde{R}_{2}$, i.e., a level 2 element in $\mathfrak{g}^{+}$. An ancillary element at ghost number 1 can be characterised as $\left[\left[B_{M N}, H^{b M}\right], H^{b N}\right]$, where $M N$ are symmetric and in section (the antisymmetric part vanishes due to the section constraint). Note, however, that its appearance relies on a non-vanishing $\widetilde{R}_{1}$.

The ancillary ghosts at ghost number 1 are thus characterised as doubly sectionconstrained objects constructed (through $\widetilde{R}_{1}$ ) from $\widetilde{R}_{2}$. This is unlike higher ancillary ghosts $K_{p}, p \geqslant 1$, which only need a single section-constrained index, and are obtained as $K_{p}^{b}=\left[B_{M}, H^{b}\right]$ with $B_{M}$ in $\widetilde{R}_{p+1}$. 
Let us consider the commutator of two ancillary transformations $\Sigma$ and $\Sigma^{\prime}$. We write

$$
\Sigma=-\left[\left[\left[\xi^{\sharp}, \partial_{M} \partial_{N} \eta^{\sharp}\right], H^{b M}\right], H^{b N}\right]=\left[\left[B_{M N}, H^{b M}\right], H^{b N}\right] .
$$

Let $V \in \widetilde{R}_{2}$ at $(p, q)=(2,2)$ and let $s_{M N P}$ be a tensor whose all indices are in section. Then $s_{M N P}\left[\left[\left[V, H^{b M}\right], H^{b N}\right], H^{b P}\right]=0$. This follows from the observation that $V$ contains irreducible representations $\overline{R\left(2 \lambda-\alpha_{i}-\delta\right)}$ with $\delta$ in the positive root lattice. The consecutive commutators with $H^{\mathrm{b}}$ contribute $R(3 \lambda)$. The result must be in $R(\lambda)$, but this representation is not part of $R(3 \lambda) \otimes \overline{R\left(2 \lambda-\alpha_{i}-\delta\right)}$, since $R(\lambda) \otimes R\left(2 \lambda-\alpha_{i}-\delta\right)$ does not contain $R(3 \lambda)$. If we then commute two ancillary generators, we immediately get

$$
\left[\Sigma, \Sigma^{\prime}\right]=\left[\left[\left[\Sigma, B_{M N}^{\prime}\right], H^{b M}\right], H^{b N}\right]=\left[\left[\left[B_{M N}, \Sigma^{\prime}\right], H^{b M}\right], H^{b N}\right]
$$

which again is of the same type.

The same two-derivative form of the ancillary transformations is also obtained by considering reducibility. In the absence of ancillary transformations we had $\mathscr{L}_{K^{b}} V=0$, where $K$ is an ancillary ghost with ghost number 2 , obtained as $K^{b}=\left[B_{M}, H^{b M}\right]$ with $B_{M}$ in $\widetilde{R}_{2}$ (for higher ancillary ghosts the statement is trivial). Inserting this into the generalised diffeomorphisms gives

$$
\mathscr{L}_{K^{b}} V+\left[(d K)^{b}, V\right]=0,
$$

so that now $K$ represents reducibility involving both the generalised diffeomorphisms and the ancillary transformations. Note that this consideration also gives ancillary ghosts at ghost number 1 constructed with two section-constrained indices from $\widetilde{R}_{2}$.

\section{Dynamics}

The remainder (2.14) in the transformation of the part $\mathscr{L}_{0}$ of the action contains the tensor $S$ and arises only in situations when ancillary transformations are present. A candidate term, that is non-zero only in these cases, is suggested by the "new" invariant tensor $\ell$, occurring as structure constants in $S$ in eq. (3.6), and projecting (with some weights) on the irreducible modules in $\widetilde{R}_{1}$ :

$$
\mathscr{L}_{1}=\eta^{\alpha \gamma} G^{M P} \ell_{\alpha M}{ }^{\beta N} \Pi_{P \beta} \Pi_{N \gamma} .
$$

It has the right indices to contract a bilinear in $\Pi$ (together with an inverse metric to match the weights) and vanishes in the absence of ancillary transformations. A straight-forward calculation gives at hand that the inhomogeneous transformation cancels the one of $\mathscr{L}_{0}$. The calculation relies on the behaviour of $\ell$ under the involution,

$$
\ell_{\alpha M}{ }^{\beta N} t^{\alpha} \otimes t_{\beta}=G_{M P} G^{N Q} \ell_{\beta Q}{ }^{\alpha P} t_{\alpha}^{\star} \otimes t^{\star \beta} .
$$

This property implies (thanks to $\Pi_{M \alpha} t^{\alpha}=\Pi_{M \alpha} t^{\star \alpha}$ ) that the tensor contracting the $\Pi$ 's in $\mathscr{L}_{1}$ is effectively symmetric under $P \beta \leftrightarrow N \gamma$. The inhomogeneous part of the variation becomes

$$
\Delta_{\xi} \mathscr{L}_{1}=2 \ell_{\alpha M}{ }^{\beta N} G^{M P}\left(t^{\alpha}{ }_{Q}^{R} \Pi_{P \beta} \partial_{N} \partial_{R} \xi^{Q}+t_{\beta Q}{ }^{R} \Pi_{N}{ }^{\alpha} \partial_{P} \partial_{R} \xi^{Q}\right)
$$


The first term gives the $S$ tensor, thanks to the identity (3.10), and precisely cancels $\Delta_{\xi} \mathscr{L}_{0}$ in eq. (2.14). In the second term, we need a "new" identity involving the section condition in the indices $N R$. Consider the invariant tensor occurring in the second term,

$$
m_{\alpha M, Q}{ }^{N R} \equiv \ell_{\alpha M}{ }^{\beta\langle N} t_{\beta Q}^{R\rangle}+\ell_{\alpha M}{ }^{\beta\{N} t_{\beta Q}{ }^{R\}} .
$$

The lower indices are in sums of $R(\lambda) \otimes R(\lambda-\beta)$, where again the $\beta$ 's are lowest roots at level 2 or higher. This tensor product contains neither $R(2 \lambda)$ nor $R\left(2 \lambda-\alpha_{i}\right)\left(\lambda_{i}=1\right)$, so $m$ is identically 0 . The second term in the variation above vanishes.

The Lagrangian $\mathscr{L}=\mathscr{L}_{0}+\mathscr{L}_{1}$ thus encodes the complete dynamics for all extended geometries with finite-dimensional structure group.

It is encouraging that the structure constants of the THA can be used to construct an invariant Lagrangian. It seems quite clear that it will be possible to form the Lagrangian as a combination of invariant contractions bilinear in the projections of $d G G^{-1}$ on the torsion modules at level -1 in $W(\mathfrak{g})$. A task in continued investigations will be to see if this specific combination has a natural origin in the superalgebra. However, $d G G^{-1}$ does not transform as a connection, but as the symmetrised (with respect to the involution) part of a connection. An alternative, but equivalent construction (see ref. [40] for a discussion) is based on the Weitzenböck connection $d E E^{-1}$, where $E$ is a generalised vielbein parametrising the coset $G / K$. The torsion part of this connection does transform as a tensor, but then a specific combination of terms will instead be dictated by invariance under local $K$ transformations, in complete analogy with the construction of the action in the teleparallel formulation of gravity. It remains to be seen which is the most efficient way of formulating the dynamics in terms of the superalgebra.

\section{$6 \quad L_{\infty}$ algebra}

The infinite tower of ghosts in exceptional field theory was first described in ref. [30], then without the introduction of ancillary ghosts. Ref. [4] showed how the generalised diffeomorphisms in exceptional field theory are constructed using a Borcherds superalgebra, and this was generalised to the framework of extended geometry in refs. [2,3]. The $L_{\infty}$ algebra for double geometry was constructed in refs. [51, 54, 55]. In this case, there are no ancillary ghosts, and the algebra stops at ghost number 2 and a 3-bracket. This corresponds to the Borcherds superalgebra being finite-dimensional. In ref. [3] the picture of ref. [30] was refined by the introduction of ancillary ghosts (with ghost number $>1$ ) and the construction of the $L_{\infty}$ algebra (2.16), which completely encodes the gauge structure of extended geometry in the absence of ancillary gauge transformations. We will now demonstrate that the THA $S$ is the correct underlying algebraic structure in the more general case.

As discussed in section 3 , the THA $S$ essentially agrees with the Borcherds superalgebra $\mathscr{B}\left(\mathfrak{g}^{+}\right)$at positive levels $p-q$. A few differences arise that are relevant for the ghost structure, i.e., for the $L_{\infty}$ algebra. The first is the presence of $\widetilde{R}_{1}$, which makes it possible to address the issue with ancillary ghosts at ghost number 1 . The second is the presence of $T^{\sharp}$ (but not $f_{0}$ ), which is where these ancillary ghosts actually reside. Table 5 shows the 


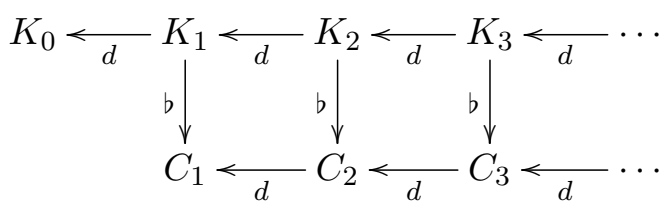

Table 5. The typical structure of the action of the 1-bracket between the ghost modules, with ancillary ghosts appearing from level $p=0$.

generic structure of the ghosts, with arrows showing the action of the nilpotent "derivative", the $L_{\infty}$ 1-bracket.

The only difference from the construction with the Borcherds superalgebra in ref. [3] is that we now may have ghosts in $K_{0}=k$. When formulating the $L_{\infty}$ brackets as derived brackets based on $S$ instead of $\mathscr{B}$, we can to a large extent rely on the previous calculations, and ask how they are modified by $k$.

The ghosts come in two kinds: the "ordinary" or non-ancillary ones at $p>0$ and $q=0$, and the ancillary ones at $p \geqslant p_{0}$ and $q=1$, where $\widetilde{R}_{p_{0}+1}$ is the lowest occurrence of an $\widetilde{R}_{p}$. In $\mathscr{B}$, we had $p_{0} \geqslant 1$. For the cases presently under consideration, with finite-dimensional $\mathfrak{g}$, the corresponding limit in $S$ is $p_{0} \geqslant 0$. (For infinite-dimensional $\mathfrak{g}$, ancillary fields may appear at lower $p$, see the discussion in section 7.) As for $\mathscr{B}$, the non-ancillary ghosts are collectively denoted $C$ and the ancillary ones $K$, but now seen as elements in $S$. The ancillary ghosts are defined as in section 4 .

We will not derive a full set of brackets and prove all identities, but content ourselves with some low brackets, together with conjectures on the general structure.

\subsection{Definitions and identities}

In ref. [3], we needed to assume the closure of the generalised diffeomorphisms, on the form $\mathscr{L}_{C} \mathscr{L}_{C}=-\frac{1}{2} \mathscr{L}_{\mathscr{L}_{C} C}$. The absence of ancillary transformations had to be assumed, and did not follow from the content of the Borcherds superalgebra - even in situations with ancillary transformations, there was no support for them in the superalgebra. This prevented us from treating cases with ancillary transformations.

As in ref. [2], the generalised Lie derivative, with one non-ancillary element $A$ as parameter, acting on another non-ancillary element $B$, both of arbitrary statistics, was defined as

$$
\mathscr{L}_{A} B=\delta_{p_{A}, 1}\left(\left[\left[A, H^{b M}\right], \partial_{M} B\right]+(-1)^{|B|}\left[\left[\partial_{M} A^{\sharp}, H^{b M}\right], B\right]\right) .
$$

This expressions (now adapted to our notation for the basis elements of $S$ ) still holds, since it does not involve ancillary elements, and thus derives from isomorphic subalgebras of $\mathscr{B}$ and $S$. Let $c=C_{1} \in R_{1}$ be non-ancillary ghost at ghost number 1 . In the presence of ancillary transformations, we have (see eq. (4.8))

$$
\mathscr{L}_{c} \mathscr{L}_{c} A=-\frac{1}{2} \mathscr{L}_{\mathscr{L}_{c} c} A+\frac{1}{2}\left[A, X_{c}^{b} c\right]
$$


where

$$
X_{a}^{b} B=-\frac{1}{2}(-1)^{|B|}\left[\left[\left[\partial_{M} \partial_{N} a^{\sharp}, B^{\sharp}\right], H^{b M}\right], H^{b N}\right],
$$

for $a$ at $(p, q)=(1,0)$ and $B$ at $q=0$.

The 1-bracket contains a horizontal part and a vertical part. The vertical part is obtained from the lowering operator,

$$
A^{b}=-\left[A, e_{0}\right]
$$

The horizontal part is defined as

$$
d A=\left[\partial_{M} A^{\sharp}, H^{b M}\right]
$$

for any element $A$ such that $A^{b}=0$, i.e., for the lower element in a pair. It is then extended to the upper elements in the pairs (the ancillary ghosts) by $(d K)^{b}=-d K^{b}$ for $K$ such that $K^{\sharp}=0$. Then $d+b$ acts as a nilpotent 1-bracket. Also the generalised Lie derivative is extended to elements $K$ at height 1 by $\left(\mathscr{L}_{a} K\right)^{b}=-\mathscr{L}_{a} K^{b}$.

The generalised Lie derivative has the usual reducibility $\mathscr{L}_{d C_{2}} A=0$, where $C_{2}$ is an element at $(p, q)=(0,2)$. In addition, there is a reducibility coming from parameters $\llbracket K_{1} \rrbracket=d K_{1}+K_{1}^{b}$, where $K_{1}$ is an ancillary element at $(p, q)=(1,1)$. The corresponding identity,

$$
\mathscr{L}_{K_{1}^{b}} A-\left[A,\left(d K_{1}\right)^{b}\right]=0,
$$

was derived in section 4 .

The 2-derivative expression $X_{a} B$ of eq. (6.3) appeared already in the previous construction with the Borcherds superalgebra [3], however only for $B$ at $p \geqslant 2$. There it arose from the non-covariance of the derivative $d$ as

$$
\left(d \mathscr{L}_{a}+\mathscr{L}_{a} d\right) B=-X_{a}^{b} B
$$

while we obtained it above from the commutator of two generalised derivatives. (For a discussion of the connection between non-covariance and the appearance of ancillary transformations, see ref. [40].) We need to check that eq. (6.7) still holds when the derivative acts on an ancillary ghost $K_{1}$, i.e., from $(p, q)=(1,1)$ to $(0,1)$. This is an issue, since the corresponding (lowered) action from $(p, q)=(1,0)$ to $(0,0)$ is never covariant, not even in the absence of ancillary transformations. We have

$$
\left(d \mathscr{L}_{a}+\mathscr{L}_{a} d\right) b=-X_{a}^{b} b+Y_{a} b,
$$

where the non-ancillary contribution is

$$
Y_{a} b=-(-1)^{|b|} \partial_{M} \partial_{N} a^{P} b^{N} t^{\alpha}{ }_{M}^{P} T_{\alpha} .
$$

If $b$ is ancillary, i.e., $b=\left[\beta_{M}, H^{\mathrm{b} M}\right]$ for $\beta_{M}$ in $\widetilde{R}_{2}, Y_{a} b$ vanishes thanks to the antisymmetric section constraint. The identity (6.7) can be used on all ghosts. 
In ref. [3], identities for commutators between derivatives, generalised Lie derivatives and ancillary operators were derived, and used in order to check the identities for the $L_{\infty}$ brackets. We only need to consider modifications involving the presence of $\widetilde{R}_{1}$ and the ghost $k$.

The entities involved are the derivative $d$, the generalised Lie derivative $\mathscr{L}_{a} B$, the two-derivative ancillary element $X_{a} B$ of eq. (6.3) and the one-derivative ancillary element $R(A, B)$ defined by

$$
R^{b}(A, B)=(-1)^{|B|} \frac{p_{B} \partial_{M}^{(A)}-p_{A} \partial_{M}^{(B)}}{p_{A}+p_{B}}\left[\left[A^{\sharp}, B^{\sharp}\right], H^{b M}\right]^{b} .
$$

The latter expression roughly indicates the deviation of $d$ from being a derivation. Let $a, b$ and $A, B$ be elements at $q=0$ and let $p_{a}=p_{b}=1, p_{A}, p_{B}>1$. Then,

$$
\begin{aligned}
d[a, b] & =\mathscr{L}_{a} b-(-1)^{|a||b|} \mathscr{L}_{b} a-R^{b}(a, b), \\
d[a, B] & =[a, d B]+\mathscr{L}_{a} B-R^{b}(a, B), \\
d[A, B] & =[A, d B]+(-1)^{|B|}[d A, B]-R^{b}(A, B) .
\end{aligned}
$$

In addition it is straightforward to derive

$$
d\left[a, \alpha^{b}\right]=\mathscr{L}_{a} \alpha^{b}-R^{b}\left(a, \alpha^{b}\right),
$$

where $a$ is an element at $(p, q)=(1,0)$ and $\alpha$ is an ancillary element in the adjoint at $(p, q)=(0,1)$.

We will not give a full list of identities. Most of them, except for very low ghost number, coincide with the ones in ref. [3]. From the expression (6.7) it immediately follows that

$$
\left(d X_{a}+X_{a} d\right) B=0 .
$$

The explicit expression for $R(a, b)$ gives

$$
d R(a, b)=X_{a} b-(-1)^{|a||b|} X_{b} a .
$$

Another identity at $(p, q)=(0,1)$ is

$$
\mathscr{L}_{c} X_{c} c+X_{c} \mathscr{L}_{c} c=-\frac{1}{2} X_{\mathscr{L}_{c} c} c+\frac{1}{2} R\left(c, X_{c}^{b} c\right)
$$

which can be proven by comparing the explicit expressions. It generalised the corresponding relation " $\mathscr{L}_{c} X_{c} A+X_{c} \mathscr{L}_{c} A=-\frac{1}{2} X_{\mathscr{L}_{c} c} A$ " in the Borcherds case.

\subsection{Some $L_{\infty}$ brackets}

When we truncate to the ghost sector, we in addition postulate that the 1-bracket annihilates the lowest ghosts. The 1-bracket is

$$
\begin{aligned}
\llbracket c \rrbracket & =0, \\
\llbracket C_{p} \rrbracket & =d C_{p}, \quad p \geqslant 2, \\
\llbracket k \rrbracket & =0, \\
\llbracket K_{p} \rrbracket & =d K_{p}+K_{p}^{b}, \quad p \geqslant 1,
\end{aligned}
$$


where we denote the ghost number 1 non-ancillary ghost $c=C_{1}$ and the ancillary one $k=K_{0}$ (the subscript is the $p$ eigenvalue, not the ghost number). This is depicted in table 5 .

Let us start with the 2-brackets between elements at ghost number 1 . We let $c \in R_{(1,0)}$ and $k \in R_{(0,1)}$. The 1-bracket by definition annihilates $c+k, \llbracket c+k \rrbracket=0$. The 2-brackets reflect the commutators between transformations, and we have, using the form of the ancillary transformations derived in section 4 ,

$$
\begin{aligned}
\llbracket c, c \rrbracket & =\mathscr{L}_{c} c+X_{c} c, \\
\llbracket c, k \rrbracket_{0} & =\mathscr{L}_{c} k, \\
\llbracket k, k \rrbracket & =-\left[k, k^{b}\right]
\end{aligned}
$$

The 2-bracket identities are of course trivially satisfied. The second of these brackets have been equipped with subscript 0 , since it will be modified. When the ghost $k$ is present, the bracket $\llbracket c, k \rrbracket$ is not uniquely determined by the commutator of a generalised Lie derivative and an ancillary transformation. The action of the commutator on e.g. a vector is of course unique, but the corresponding set of parameters is not. We can choose to add a trivial term proportional to

$$
\llbracket[c, k]^{b \sharp} \rrbracket=-\left(d\left[c, k^{b}\right]\right)^{\sharp}+\left[c, k^{b}\right]=\mathscr{L}_{c} k+R\left(c, k^{b}\right)+\left[c, k^{b}\right],
$$

where we have used eq. (6.12) in the last step, representing a vanishing tranformation due to reducibility. The operation $b \sharp$ ensures that the parameter lies in $R_{1}$ at height 1 , and not in $\widetilde{R}_{1}$. The choice made here will have repercussions for the brackets containing higher ghost number ghosts and for higher brackets. A goal is to connect as closely as possible to the results of ref. [3], where choices for low brackets enabled us to give universal expressions for all brackets between all ghosts. In particular, we then had $\llbracket C, K \rrbracket=\frac{1}{2} \mathscr{L}_{C} K$, with a factor which contrasts with the middle equation in (6.17). It will serve our purposes to add the trivial transformation of eq. (6.18) with coefficient $-\frac{1}{2}$ to obtain

$$
\begin{aligned}
\llbracket c, c \rrbracket & =\mathscr{L}_{c} c+X_{c} c, \\
\llbracket c, k \rrbracket & =\mathscr{L}_{c} k+\frac{1}{2} \llbracket[c, k]^{b \sharp}=\frac{1}{2} \mathscr{L}_{c} k-\frac{1}{2} R\left(c, k^{b}\right)-\frac{1}{2}\left[c, k^{b}\right], \\
\llbracket k, k \rrbracket & =-\left[k, k^{b}\right]
\end{aligned}
$$

The "algebra" of eq. (6.17) is a simple choice of representative in that it contains no generalised diffeomorphisms in the commutator between a generalised diffeomorphism and an ancillary transformation. The representative in eq. (6.19), on the other hand, is not of this kind, but has other advantages.

In ref. [3], the coefficient of the $n$-brackets contained the Bernoulli number $B_{n-1}$. The vanishing of the Bernoulli numbers for odd argument $\geqslant 3$ implied the vanishing of the even brackets, starting from the 4-bracket. It would be desirable to maintain this property. This turns out to be possible, using the choice above and further choices for higher brackets.

Before completing the 2-brackets, we consider the 3 -bracket $\llbracket c, c, c \rrbracket$. The identity is

$$
\llbracket \llbracket c, c, c \rrbracket \rrbracket+2 \llbracket c, \llbracket c, c \rrbracket \rrbracket=0 .
$$


The second term is calculated as

$$
\begin{aligned}
2 \llbracket c, \llbracket c, c \rrbracket \rrbracket= & 2 \llbracket c, \mathscr{L}_{c} c+X_{c} c \rrbracket \\
= & \mathscr{L}_{c} \mathscr{L}_{c} c+\mathscr{L}_{\mathscr{L}_{c} c} \\
& +X_{c} \mathscr{L}_{c} c+X_{\mathscr{L}_{c} c} c+\mathscr{L}_{c} X_{c} c-R\left(c, X_{c}^{b} c\right)-\left[c, X_{c}^{b} c\right] .
\end{aligned}
$$

The terms at $q=0$ (the first two terms, together with the last one, coming from the modification of the bracket $\llbracket c, k \rrbracket)$ can be rewritten using the first equation in (6.11) together with eq. (6.2) as

$$
\mathscr{L}_{c} \mathscr{L}_{c} c+\mathscr{L}_{\mathscr{L}_{c} c} c-\left[c, X_{c}^{b} c\right]=-\frac{1}{3}\left(d\left[c, \mathscr{L}_{c} c\right]+R^{b}\left(c, \mathscr{L}_{c} c\right)+\left[c, X_{c}^{b} c\right]\right) .
$$

This immediately gives the full 3-bracket as

$$
\llbracket c, c, c \rrbracket=\frac{1}{3}\left\{\left[c, \mathscr{L}_{c} c\right]+R\left(c, \mathscr{L}_{c} c\right)+\left[c, X_{c} c\right]\right\}
$$

It remains to be verified that the $q=1$ part of the identity is satisfied. This demands that

$$
\frac{1}{3} d R\left(c, \mathscr{L}_{c} c\right)+\frac{1}{3} d\left(\left[c, X_{c} c\right]^{b \sharp}\right)+\mathscr{L}_{c} X_{c} c+X_{c} \mathscr{L}_{c} c+X_{\mathscr{L}_{c} c} c-R\left(c, X_{c}^{b} c\right)=0 .
$$

A short calculation, using eqs. (6.14) and (6.12), shows the $q=1$ part of the identity holds, thanks to eq. (6.15). Note that 3-bracket has the same formal expression as in eq. (2.16). In particular, the coefficients of $\left[c, \mathscr{L}_{c} c\right]$ and $\left[c, X_{c} c\right]$ are the same, and this happens only for our particular choice of representative for $\llbracket c, k \rrbracket$.

Let us now address the vanishing of the 4-bracket $\llbracket c, c, c, c \rrbracket$. The identity to be fulfilled is

$$
\llbracket \llbracket c, c, c, c \rrbracket \rrbracket+2 \llbracket c, \llbracket c, c, c \rrbracket \rrbracket+3 \llbracket c, c, \llbracket c, c \rrbracket \rrbracket=0,
$$

and we will for now only consider the $q=0$ part. Assuming that $\llbracket c, K_{1} \rrbracket$ does not contain a $q=0$ part (see below), it can be calculated as

$$
\begin{aligned}
& \llbracket \llbracket c, c, c,\left.c \rrbracket \rrbracket\right|_{q=0}+\frac{2}{3}\left[c, \mathscr{L}_{c} \mathscr{L}_{c} c+\frac{1}{2} \mathscr{L}_{\mathscr{L}_{c} c} c\right]+3 \llbracket c, c,\left.X_{c} c \rrbracket\right|_{q=0} \\
& =\llbracket \llbracket c, c, c,\left.c \rrbracket \rrbracket\right|_{q=0}+\frac{1}{3}\left[c,\left[c, X_{c}^{b} c\right]\right]+3 \llbracket c, c,\left.X_{c} c \rrbracket\right|_{q=0} .
\end{aligned}
$$

A necessary condition for the consistency of the vanishing of the 4-bracket is that $\llbracket c, c, k \rrbracket$ contains a $q=0$ part $-\frac{1}{9}\left[c,\left[c, k^{b}\right]\right]$. This can be arranged by choosing a representative by the suitable addition of a trivial term proportional to $\llbracket[c,[c, k]]^{b \sharp} \rrbracket$. It also becomes clear - by the calculations in the Borcherds case together with the ancillary term in $\mathscr{L}_{c} \mathscr{L}_{c}$ in eq. (6.2) - that this happens to all orders, and that we can reproduce the collective brackets

$$
\llbracket C^{n} \rrbracket=k_{n}\left((\operatorname{ad} C)^{n-2}\left(\mathscr{L}_{C} C+X_{C} C\right)+\sum_{i=0}^{n-3}(\operatorname{ad} C)^{i} R_{C}(\operatorname{ad} C)^{n-i-3} \mathscr{L}_{C} C\right),
$$

as before, with the new extended meaning of $X_{C} C$. 
Let us now consider brackets with one ancillary ghost. The lowest identity is

$$
\begin{aligned}
0 & =\llbracket \llbracket c, K_{1} \rrbracket \rrbracket+\llbracket c, \llbracket K_{1} \rrbracket \rrbracket \\
& =\llbracket \llbracket c, K_{1} \rrbracket \rrbracket+\llbracket c, d K_{1} \rrbracket+\llbracket c, K_{1}^{b} \rrbracket .
\end{aligned}
$$

The last two terms become, using eq. (6.19),

$$
\begin{aligned}
\llbracket c, d K_{1} \rrbracket+\llbracket c, K_{1}^{b} \rrbracket= & \mathscr{L}_{c} d K_{1}-\frac{1}{2} R\left(c, d K_{1}\right)-\frac{1}{2}\left[c,\left(d K_{1}\right)^{b}\right] \\
& +\frac{1}{2} \mathscr{L}_{c} K_{1}^{b}+\frac{1}{2} \mathscr{L}_{K_{1}^{b}} c+\frac{1}{2} X_{c} K_{1}^{b}+\frac{1}{2} X_{K_{1}^{b}} c .
\end{aligned}
$$

Using the reducibility $\mathscr{L}_{K_{1}^{\mathrm{b}}} c-\left[c,\left(d K_{1}\right)^{\mathrm{b}}\right]=0$, the height 0 terms become $-\frac{1}{2} \mathscr{L}_{c} K_{1}$, implying that

$$
\llbracket c, K_{1} \rrbracket=\frac{1}{2} \mathscr{L}_{c} K_{1},
$$

which agrees with the expression in the Borcherds case. The remaining terms at height 1 of the identity demand are

$$
\begin{aligned}
\frac{1}{2}\left(d \mathscr{L}_{c} K_{1}+\mathscr{L}_{c} d K_{1}+X_{c} K_{1}^{b}+X_{K_{1}^{b}} c-R\left(c,\left(d K_{1}\right)^{b}\right)\right. & \\
= & \frac{1}{2}\left(X_{K_{1}^{b}} c-R\left(c,\left(d K_{1}\right)^{b}\right)\right)=0 .
\end{aligned}
$$

This result is readily extended to

$$
\llbracket C, K^{\prime} \rrbracket=\frac{1}{2} \mathscr{L}_{C} K^{\prime},
$$

where $K^{\prime}=K-k$ is the $p \geqslant 1$ part of $K$, in accordance with the Borcherds case.

For the 2-brackets with two ancillary ghosts one easily obtains

$$
\begin{aligned}
\llbracket k, K^{\prime} \rrbracket & =-\frac{1}{2}\left[K^{\prime}, k^{b}\right], \\
\llbracket K^{\prime}, K^{\prime} \rrbracket & =0 .
\end{aligned}
$$

This completes the 2-brackets between all ghosts. It is possible to introduce the notation $\mathscr{M}_{K} A=-\left[A, k^{b}\right]$ and $Y_{K} A=-R\left(A, k^{b}\right)$ (in analogy with $\mathscr{L}_{C} A=\mathscr{L}_{c} A$ ), in order to write the 2-brackets collectively as

$$
\begin{aligned}
\llbracket C, C \rrbracket & =\mathscr{L}_{C} C+X_{C} C, \\
\llbracket C, K \rrbracket & =\frac{1}{2}\left(\mathscr{L}_{C} K+\mathscr{M}_{K} C+Y_{K} C\right), \\
\llbracket K, K \rrbracket & =\mathscr{M}_{K} K .
\end{aligned}
$$

It is clear that the construction works, and that there are solutions to the identities that make the higher brackets take forms close to the ones in the Borcherds case. We conjecture that all brackets reduce to the formal expressions for the Borcherds ones under $k \rightarrow 0$, that all brackets with more than two ancillary ghosts vanish, and that all even brackets above the 2-bracket vanish. 


\begin{tabular}{|c|c|c|c|}
\hline & $p=-1$ & $p=0$ & $p=1$ \\
\hline$q=2$ & & $\begin{array}{c}\pi^{\sharp} \\
1\end{array}$ & $L_{M}^{\sharp}$ \\
\hline$q=1$ & $\Phi_{0}^{\sharp M}$ & 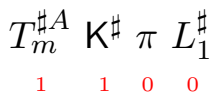 & $\begin{array}{cc}E_{M}^{\sharp} & L_{M} \\
1 & 0\end{array}$ \\
\hline$q=0$ & $\begin{array}{cc}\Phi^{M} & H^{M} \\
-1 & 0\end{array}$ & $\begin{array}{ccccc}T_{m}^{A} & \mathrm{~K} & \mathrm{~d} & L_{1} \\
0 & 0 & 0 & -1\end{array}$ & $\underset{0}{E_{M}}$ \\
\hline$q=-1$ & $\begin{array}{c}H_{-1}^{b M} \\
-1\end{array}$ & $\begin{array}{l}e_{0} \\
-1\end{array}$ & \\
\hline
\end{tabular}

Table 6. Basis elements for $S\left(\mathfrak{g}^{+}\right)$when $\mathfrak{g}$ is an affine algebra. The shifts specifying the action of $\mathrm{d}$ are given in red.

\section{Discussion}

We have given a description of the dynamics and gauge structure of extended geometry with a finite-dimensional structure group $G$ and lowest weight coordinate representation $R(-\lambda)$. The tensor hierarchy algebra $S=S\left(\mathfrak{g}^{+}\right)$plays a central rôle, in that it (unlike the corresponding Borcherds superalgebra) naturally harbours the ancillary transformations. Ingredients from the THA were also used for a description of the dynamics.

One main purpose of the paper, and its companion [1], is to find the underlying algebraic structure behind extended geometry. The THA $S$, in every respect, shows promise to contain exactly the correct information precisely when it is needed. One of the relevant aspects for the present investigation is the peculiar appearance of new elements (in the present case, a lowest weight $\mathfrak{g}^{+}$-module, starting with the generators $L_{\alpha M}$ ) along with $\mathfrak{g}^{+}$at level 0 (cf. ref. [48]). It is tempting to extrapolate to more complicated situations, especially with infinite-dimensional structure groups [42], and follow the lead given by the THA. For $G=E_{9}$, the relevant THA algebra is $S\left(E_{10}\right)$. The central part of this superalgebra (or more generally, for any affine $G$ ), decomposed into $E_{9}$ representations along the same principles as previous tables, is given in table 6. In order to construct this part of the algebra, one needs to include the generator $\mathrm{d}$ (the Virasoro generator $L_{0}$, roughly speaking) in order to have a non-degenerate Cartan matrix (bilinear form).

Here, the generators at $(p, q)=(0,0)$ are the affine generators, including $\mathrm{d}$, which is $L_{0}$, but acting with a "shift" compared to $L_{0}$, indicated in red below the generators. There is also $L_{1}$, which we have already encountered in $S\left(E_{9}\right)$. The elements at $p=-1$ and $p=1$ are (shifted) fundamentals and anti-fundamentals of $E_{9}$. It is noteworthy that now even $\widetilde{R}_{0}$ is non-empty, and consists of a singlet. We have seen that the presence of $\widetilde{R}_{p}$ indicates ancillary ghosts at ghost number $p$, compensating for an apparent failure in the $(p+1)$ - 
bracket. It will be interesting to see what this means for the 1-bracket in $E_{9}$ geometry. In the $E_{9}$ case, or more generally in the affine case, including the geometrisation of the Geroch group, this seems to point at the appearance of ancillary fields already at ghost number 0 , represented by constrained fields in the unshifted fundamental representation at $(p, q)=(-1,1)$. This would corroborate and provide an algebraic basis for the results of ref. [43], and will be the subject of a forthcoming study.

The main purpose of the invention of THA's [6] was to have the embedding tensor at level -1 . This representation $(\Phi)$ together with a "fundamental" $(H)$ form the representations in which torsion comes in extended geometry. It is likely that the ghost sector can be complemented with dynamical fields at level 0, "torsion" antifields at level -1 , and further higher antifields corresponding to torsion Bianchi identities etc. ("syzygies") in the algebraic framework. This is of course completely beyond the reach of a Borcherds superalgebra.

The fact that the generalised Lie derivative fulfils a Leibniz rule means that the vector fields satisfy a Leibniz algebra $[56,57]$. It has recently been shown that any Leibniz algebra canonically (via a differential graded Lie algebra, or equivalently, an infinity-enhanced Leibniz algebra [58]) gives rise to an $L_{\infty}$ algebra [59]. It would be interesting to compare the $L_{\infty}$ algebra constructed in that way with the one presented here, not least since in the application to gauged supergravity, the relevant differential graded Lie algebra can be understood as coming from a tensor hierarchy algebra [60, 61].

In some cases, notably in the exceptional series, the THA's of $S$ type possess a nondegenerate invariant bilinear form $[6,48]$, which is invariant under the algebra but not centered around level 0 . For $S\left(E_{r+1}\right)$, a $\mathfrak{g}$-module at $(p, q)$ in the double grading is paired with the conjugate module at $(9-r-p, 1-q)$. Given an involution on $\mathfrak{g}$, one may use it to define a "dualisation" map $\star \in \operatorname{End}\left(S\left(\mathfrak{g}^{+}\right)\right)$and, as proposed in [48], the bilinear form might be used in an action.

\section{Acknowledgments}

MC wants to thank E. Almqvist for discussions on factors of $\frac{1}{2}$. This research is supported by the Swedish Research Council, project no. 2015-04268.

Open Access. This article is distributed under the terms of the Creative Commons Attribution License (CC-BY 4.0), which permits any use, distribution and reproduction in any medium, provided the original author(s) and source are credited.

\section{References}

[1] M. Cederwall and J. Palmkvist, Tensor hierarchy algebras and extended geometry. Part I. Construction of the algebra, JHEP 02 (2020) 144 [arXiv:1908.08695] [INSPIRE].

[2] M. Cederwall and J. Palmkvist, Extended geometries, JHEP 02 (2018) 071 [arXiv: 1711.07694] [INSPIRE].

[3] M. Cederwall and J. Palmkvist, $L_{\infty}$ Algebras for Extended Geometry from Borcherds Superalgebras, Commun. Math. Phys. 369 (2019) 721 [arXiv: 1804.04377] [InSPIRE]. 
[4] J. Palmkvist, Exceptional geometry and Borcherds superalgebras, JHEP 11 (2015) 032 [arXiv: 1507.08828] [INSPIRE].

[5] M. Cederwall and J. Palmkvist, Superalgebras, constraints and partition functions, JHEP 08 (2015) 036 [arXiv: 1503.06215] [INSPIRE].

[6] J. Palmkvist, The tensor hierarchy algebra, J. Math. Phys. 55 (2014) 011701 [arXiv: 1305.0018] [INSPIRE].

[7] L. Carbone, M. Cederwall and J. Palmkvist, Generators and relations for Lie superalgebras of Cartan type, J. Phys. A 52 (2019) 055203 [arXiv: 1802.05767] [inSPIRE].

[8] A.A. Tseytlin, Duality symmetric closed string theory and interacting chiral scalars, Nucl. Phys. B 350 (1991) 395 [InSPIRE].

[9] W. Siegel, Two vierbein formalism for string inspired axionic gravity, Phys. Rev. D 47 (1993) 5453 [hep-th/9302036] [INSPIRE].

[10] W. Siegel, Manifest duality in low-energy superstrings, in International Conference on Strings 93, Berkeley, California, 24-29 May 1993, pp. 353-363 (1993) [hep-th/9308133] [INSPIRE].

[11] N. Hitchin, Lectures on generalized geometry, arXiv:1008.0973 [INSPIRE].

[12] C.M. Hull, A Geometry for non-geometric string backgrounds, JHEP 10 (2005) 065 [hep-th/0406102] [INSPIRE].

[13] C.M. Hull, Doubled Geometry and T-Folds, JHEP 07 (2007) 080 [hep-th/0605149] [INSPIRE].

[14] C. Hull and B. Zwiebach, Double Field Theory, JHEP 09 (2009) 099 [arXiv:0904.4664] [INSPIRE].

[15] O. Hohm, C. Hull and B. Zwiebach, Background independent action for double field theory, JHEP 07 (2010) 016 [arXiv: 1003.5027] [inSPIRE].

[16] O. Hohm, C. Hull and B. Zwiebach, Generalized metric formulation of double field theory, JHEP 08 (2010) 008 [arXiv: 1006.4823] [INSPIRE].

[17] I. Jeon, K. Lee, J.-H. Park and Y. Suh, Stringy Unification of Type IIA and IIB Supergravities under $N=2 D=10$ Supersymmetric Double Field Theory, Phys. Lett. B 723 (2013) 245 [arXiv:1210.5078] [INSPIRE].

[18] J.-H. Park, Comments on double field theory and diffeomorphisms, JHEP 06 (2013) 098 [arXiv: 1304.5946] [INSPIRE].

[19] D.S. Berman, M. Cederwall and M.J. Perry, Global aspects of double geometry, JHEP 09 (2014) 066 [arXiv:1401.1311] [INSPIRE].

[20] M. Cederwall, The geometry behind double geometry, JHEP 09 (2014) 070 [arXiv:1402.2513] [INSPIRE].

[21] M. Cederwall, T-duality and non-geometric solutions from double geometry, Fortsch. Phys. 62 (2014) 942 [arXiv: 1409.4463] [INSPIRE].

[22] M. Cederwall, Double supergeometry, JHEP 06 (2016) 155 [arXiv:1603.04684] [INSPIRE].

[23] C.M. Hull, Generalised Geometry for M-theory, JHEP 07 (2007) 079 [hep-th/0701203] [INSPIRE].

[24] P. Pires Pacheco and D. Waldram, M-theory, exceptional generalised geometry and superpotentials, JHEP 09 (2008) 123 [arXiv:0804.1362] [INSPIRE]. 
[25] C. Hillmann, $E_{7(7)}$ and $d=11$ supergravity, Ph.D. Thesis, Humboldt-Universität zu Berlin (2008) [arXiv:0902.1509] [INSPIRE].

[26] D.S. Berman and M.J. Perry, Generalized Geometry and M-theory, JHEP 06 (2011) 074 [arXiv: 1008.1763] [INSPIRE].

[27] D.S. Berman, H. Godazgar and M.J. Perry, $\mathrm{SO}(5,5)$ duality in M-theory and generalized geometry, Phys. Lett. B 700 (2011) 65 [arXiv: 1103.5733] [INSPIRE].

[28] A. Coimbra, C. Strickland-Constable and D. Waldram, $E_{d(d)} \times \mathbb{R}^{+}$generalised geometry, connections and M-theory, JHEP 02 (2014) 054 [arXiv:1112.3989] [INSPIRE].

[29] A. Coimbra, C. Strickland-Constable and D. Waldram, Supergravity as Generalised Geometry II: $E_{d(d)} \times \mathbb{R}^{+}$and M-theory, JHEP 03 (2014) 019 [arXiv:1212.1586] [INSPIRE].

[30] D.S. Berman, M. Cederwall, A. Kleinschmidt and D.C. Thompson, The gauge structure of generalised diffeomorphisms, JHEP 01 (2013) 064 [arXiv:1208.5884] [INSPIRE].

[31] J.-H. Park and Y. Suh, U-geometry: SL(5), JHEP 04 (2013) 147 [Erratum ibid. 11 (2013) 210] [arXiv:1302.1652] [INSPIRE].

[32] M. Cederwall, J. Edlund and A. Karlsson, Exceptional geometry and tensor fields, JHEP 07 (2013) 028 [arXiv: 1302.6736] [INSPIRE].

[33] M. Cederwall, Non-gravitational exceptional supermultiplets, JHEP 07 (2013) 025 [arXiv: 1302.6737] [INSPIRE].

[34] G. Aldazabal, M. Graña, D. Marqués and J.A. Rosabal, Extended geometry and gauged maximal supergravity, JHEP 06 (2013) 046 [arXiv: 1302.5419] [INSPIRE].

[35] O. Hohm and H. Samtleben, Exceptional Form of D=11 Supergravity, Phys. Rev. Lett. 111 (2013) 231601 [arXiv: 1308.1673] [inSPIRE].

[36] C.D.A. Blair, E. Malek and J.-H. Park, M-theory and Type IIB from a Duality Manifest Action, JHEP 01 (2014) 172 [arXiv:1311.5109] [INSPIRE].

[37] O. Hohm and H. Samtleben, Exceptional Field Theory I: $E_{6(6)}$ covariant Form of M-theory and Type IIB, Phys. Rev. D 89 (2014) 066016 [arXiv:1312.0614] [INSPIRE].

[38] O. Hohm and H. Samtleben, Exceptional field theory. II. E 7(7) , Phys. Rev. D 89 (2014) 066017 [arXiv: 1312.4542] [INSPIRE].

[39] O. Hohm and H. Samtleben, Exceptional field theory. III. E8(8), Phys. Rev. D 90 (2014) 066002 [arXiv: 1406.3348 ] [INSPIRE].

[40] M. Cederwall and J.A. Rosabal, E 8 geometry, JHEP 07 (2015) 007 [arXiv: 1504.04843] [INSPIRE].

[41] D. Butter, H. Samtleben and E. Sezgin, $E_{7(7)}$ Exceptional Field Theory in Superspace, JHEP 01 (2019) 087 [arXiv: 1811.00038] [INSPIRE].

[42] G. Bossard, M. Cederwall, A. Kleinschmidt, J. Palmkvist and H. Samtleben, Generalized diffeomorphisms for $E_{9}$, Phys. Rev. D 96 (2017) 106022 [arXiv:1708.08936] [InSPIRE].

[43] G. Bossard, F. Ciceri, G. Inverso, A. Kleinschmidt and H. Samtleben, E9 exceptional field theory. Part I. The potential, JHEP 03 (2019) 089 [arXiv: 1811.04088] [InSPIRE].

[44] G. Bossard, A. Kleinschmidt and E. Sezgin, On supersymmetric $E_{11}$ exceptional field theory, JHEP 10 (2019) 165 [arXiv:1907.02080] [INSPIRE]. 
[45] T. Damour, M. Henneaux and H. Nicolai, $E_{10}$ and a 'small tension expansion' of M-theory, Phys. Rev. Lett. 89 (2002) 221601 [hep-th/0207267] [INSPIRE].

[46] P.C. West, $E_{11}$ and M-theory, Class. Quant. Grav. 18 (2001) 4443 [hep-th/0104081] [INSPIRE].

[47] O. Hohm and H. Samtleben, U-duality covariant gravity, JHEP 09 (2013) 080 [arXiv: 1307.0509] [INSPIRE].

[48] G. Bossard, A. Kleinschmidt, J. Palmkvist, C.N. Pope and E. Sezgin, Beyond E 11 $_{1}$, JHEP 05 (2017) 020 [arXiv: 1703.01305] [INSPIRE].

[49] T. Lada and J. Stasheff, Introduction to SH Lie algebras for physicists, Int. J. Theor. Phys. 32 (1993) 1087 [hep-th/9209099] [INSPIRE].

[50] B. Zwiebach, Closed string field theory: Quantum action and the B-V master equation, Nucl. Phys. B 390 (1993) 33 [hep-th/9206084] [INSPIRE].

[51] O. Hohm and B. Zwiebach, $L_{\infty}$ Algebras and Field Theory, Fortsch. Phys. 65 (2017) 1700014 [arXiv: 1701.08824$]$ [INSPIRE].

[52] D. Roytenberg and A. Weinstein, Courant algebroids and strongly homotopy Lie algebras, math/9802118.

[53] V.G. Kac, Lie Superalgebras, Adv. Math. 26 (1977) 8 [inSPIRE].

[54] A. Deser and C. Sämann, Extended Riemannian Geometry I: Local Double Field Theory, arXiv: 1611.02772 [INSPIRE].

[55] A. Deser and C. Sämann, Derived Brackets and Symmetries in Generalized Geometry and Double Field Theory, PoS(CORFU2017)141 (2018) [arXiv: 1803.01659] [INSPIRE].

[56] O. Hohm and H. Samtleben, Leibniz-Chern-Simons Theory and Phases of Exceptional Field Theory, Commun. Math. Phys. 369 (2019) 1055 [arXiv:1805.03220] [InSPIRE].

[57] O. Hohm and H. Samtleben, Higher Gauge Structures in Double and Exceptional Field Theory, Fortsch. Phys. 67 (2019) 1910008 [arXiv: 1903.02821] [InSPIRE].

[58] R. Bonezzi and O. Hohm, Leibniz Gauge Theories and Infinity Structures, arXiv:1904.11036 [INSPIRE].

[59] S. Lavau and J. Palmkvist, Infinity-enhancing of Leibniz algebras, arXiv:1907.05752 [INSPIRE].

[60] J. Greitz, P. Howe and J. Palmkvist, The tensor hierarchy simplified, Class. Quant. Grav. 31 (2014) 087001 [arXiv:1308.4972] [INSPIRE].

[61] S. Lavau, Tensor hierarchies and Leibniz algebras, J. Geom. Phys. 144 (2019) 147 [arXiv: 1708.07068] [INSPIRE]. 